\title{
Taeniosis/Neurocysticercosis Control as a Medical Problem-A Discussion Paper
}

\author{
Zbigniew Pawlowski \\ Medical University of Poznan, Poznan, Poland \\ Email: zpawlows@ump.edu.pl
}

Received 27 March 2016; accepted 13 May 2016; published 16 May 2016

Copyright (C) 2016 by author and Scientific Research Publishing Inc.

This work is licensed under the Creative Commons Attribution International License (CC BY). http://creativecommons.org/licenses/by/4.0/

(c) () Open Access

\begin{abstract}
Taenia solium taeniosis is a zoonosis transmitted by cysticerci from pigs. Neurocysticercosis is a fecal-borne infection spread exclusively by $T$. solium eggs in a tapeworm carrier or around. Neurocysticercosis is frequently fatal and debilitating disease and a common cause of late epilepsy. Taeniosis/neurocysticercosis (t/nc) can be prevented both by control of meat-borne zoonosis, which is a traditional responsibility of veterinarians, and by chemotherapy of human taeniosis, which is usually underestimated by medical profession. Elimination of $T$. solium taenosis is not only the matter of organized control projects but also of routine, daily medical and veterinary services. In order to promote more effective control by treatment of human $T$. solium carriers the following suggestions are proposed to be discussed: 1) accept that leaving untreated any detected human carrier of $T$. solium taeniosis, the only source of neurocysticercosis, is a medical error. This statement concerns both clinical settings and field interventions; 2) accept as the clinical and public health standards a search for $T$. solium carrier in any possible $t / n c$ foci and treat both detected and suspected cases of taeniosis; 3 ) make the diagnosis and treatment of $T$. solium infections in humans widely accessible and possibly free; 4) improve easy applicable tools detecting $T$. solium taeniosis; 5) make modern and traditional taenicides available. There is a need to improve the stability and efficacy of niclosamide and elaborate an instruction how to avoid neurological sideeffects of praziquantel; 6) include prevention of $t / n c$ into professional training, especially in mental health, public health and primary health care professions; 7) intensify preventive education adapted to the local endemic situation.
\end{abstract}

\section{Keywords}

Taenia solium, Taeniosis, Neurocysticercosis, Preventable Epilepsy, Health Education, Control

\section{Introduction}

The control of Taenia solium infection is justified exclusively by severe pathology caused by neurocysticercosis 
in humans. Therefore from the medical point of view, it would be more appropriate to use the term "the control of taeniosis/neurocysticercosis" [ $\mathrm{t} / \mathrm{nc}$ ] [1], rather than the traditional "control of taeniosis/cysticercosis" [ $\mathrm{t} / \mathrm{c}]$ or simply "control of cysticercosis". The formal change of the term, already introduced by WHO [2], would strengthen the clinical justification of the control and also turn the attention to the fecal-oral way of transmission of neurocysticercosis in or around a Taenia solium carrier. It also would convince the public health authorities that the control of $\mathrm{t} / \mathrm{nc}$ is not only a veterinary problem. The paper wants to discuss and support that view.

\section{Zoonotic Stigma in Practicing the t/nc Control}

Historically, neurocysticercosis was common in central Europe in mid-XIX century. It was found in 2\% of postmortem examinations in Berlin [3]. Since the 1850s, due to experiments of Kuechenmeister and van Beneden, it is well known that Taenia solium infections is a zoonosis, transmitted between humans and pigs. In those days the treatment of taeniosis in humans was not very efficient and might be risky, being sporadically fatal in treated patients. Therefore major control measures were directed towards the prevention of spreading this infection to humans by avoiding the consumption of raw pork and obligatory meat inspection. By the way, the routine meat inspection was first used for the control of trichinellosis, which was also very common in Germany in XIXth century [3]. That approach has reduced t/nc in Central Europe but does not work in endemic developing countries nowadays. In spite of the introduction of taenicides, in the 1960s, being effective and relatively safe, the zoonotic stigma has remained and the control of $\mathrm{t} / \mathrm{nc}$ has continued to be directed mainly against infections in pigs and is organized usually by veterinarians and veterinary departments [2]. Since the XIXth century is being known that neurocysticercosis is a fecal-borne infections, spreading in and around a human tapeworm carrier. However, the use of modern taenicides as a control measure still does not have a high priority, even nowadays, although it kills the parasite directly in a carrier of the infection [4].

\section{The Control of $t / n c$ Has Been Formally Accepted as Neglected with Much Delay}

There are three milestones in the history of $\mathrm{t} / \mathrm{nc}$ control:

1) The International Workshop on Present State of Knowledge and Perspectives in Cysticercosis, organized by the Mexican parasitologists in San Miguel de Allende in 1981 first paid an attention to neurocysticercosis, being as prevalent in the developing countries as it was in Europe in XIXth century [5]. This workshop, held over 30 years ago, has promoted neurocysticercosis as an important public health problem and has initiated an international cooperation in its control.

2) In 1993 the view was expressed that the t/nc control is theoretically possible and taeniosis/cysticercosis has been accepted by International Task Force on Disease Eradication as the one of six eradicable parasitoses [6] [7]. However, in practice this idea proved to be hard of being implemented as the transmission occurs too strongly bound to the environment of humans i.e. behavior, economy and inefficient medical, veterinary and public health services. Various control projects have been used since that time but positive information on lowering the prevalence of t/nc is scarce and come from Mexico, Ecuador, Peru and some Asian countries [8]-[11].

3) In spite of several meetings already held (listed in [12]) WHO has formally included the control of t/nc into the program of the Neglected Tropical Diseases Program as late as in 2007, deliberating a long time "which way to proceed?” However, the control of t/nc has not been included among 10 parasitic diseases discussed at the London meeting in 2012, neither at the meeting “United to Combat NTD’s in Paris in 2014 [13]. Both WHO meetings in 2009 and 2014 has been oriented to a zoonotic rather than to a human fecal-borne transmissions [12] [14]. Only recently the control of t/nc has been reactivated and a road map open for discussion accepted [2]. Problem of $T$. solium elimination is not only in developing countries. Control of $\mathrm{t} / \mathrm{nc}$ was mentioned as neglected also in the territory of USA, where fecal-borne transmission dominates and local zoonotic transmission is negligible [15] [16].

\section{Using New Diagnostic and Chemotherapy Tools Is Not Satisfactory}

During the last two decades enormous progress had been made in finding new control tools although nothing has been perfect [17] [18]. The coproantigen tests using ELISA or PCR technologies, are hardly available where might be needed. Tests for taeniosis using blood drops for finding Taenia antigens remain still at the laboratory desk. Much an effort has been made to differentiate various Taenia species, which is important in research but 
not in control practice as all Taenia species in humans deserve elimination, if detected. Two modern taenicides, namely niclosamide and praziquantel, have its weaknesses. Niclosamide (5 US\$ per treatment) is still too expensive for mass treatment. Moreover, some generic and/or long stored series have an efficacy much below expected 90\% [19]. Doubling the dose of niclosamide or using repeated treatment cycles are not an acceptable solution. Quality control of niclosamide efficacy is periodically necessary but is usually not done. Praziquantel is effective and cheap (0.05 - 0.10 US\$ per treatment) but being used in neurocysticercotic patients may damage cysticerci and sporadically change asymptomatic infection into a symptomatic one, a process which commonly occurs naturally. It is a matter of ethics to weight that sporadic risk against the elimination of a tapeworm carriership, continuously spreading neurocysticercosis further. The Mexicans took the risk and successfully treated population with $10 \mathrm{mg}$ of praziquantel. However, taenicides has not been included into the WHO preventive chemotherapy instructions selecting several anthelmintics useful to be distributed to populations at risk. Due to M \& B Gates financial support the possibility of elimination of cysticercosis in pigs becomes recently attractive due to the introduction of pig vaccination and/or pig-specific chemotherapy [4]. This rather costly procedure has to be carried out for several years, as long as the reservoir of tapeworms in humans, being the only source of neurocysticercosis, does not naturally disappear. One positive aspect of pig's vaccination is that free roaming pigs, hard to arrest, remain a sanitary police cleaning the human environment from fecal material containing $T$. solium eggs but do not developing infective cysticerci in pork.

\section{Discord about the Aim and the Optimal Tactics in t/nc Control Nearly Comes to an End}

The selection of the aim of the $t / n c$ control and the optimal tactics provoked several discussions for years. It is now clear that the global elimination of Taenia solium infections cannot be achieved in a given time limit, mainly due to the intensive migration of human carriers in endemic areas and outside. However, it is an urgent need to reduce locally the burden of the high prevalence of neurocysticercosis, wherever exists, to a level of negligible public health importance by "short term" interventions. As the transmission pattern differs in endemic areas any control measure, based either on chemotherapy, pig's vaccination, education, sanitation or combined is useful, if accepted, feasible and successful locally. The choice of tactics should depend on local endemic situation and realistic control possibilities. However, in all the control programs an immediate treatment of human Taenia solium carriers, as soon as they are detected, has to be included as a core part of intervention.

\section{Finding a Focality of Taenia solium Infections Makes the t/nc Control Easier}

Finding a Taenia solium carrier is not easy: clinical signs are weak, expelling tapeworm strobila may be overlooked, microscopic examination of feces has a low efficacy, coproantigen tests may not be available. Detection of the focality of the Taenia infections makes the chance of finding a tapeworm carrier much higher and also strongly increase a justification to treat whole group of suspected people in a focus [18] [20]. Over twenty years ago (!) there were two classical epidemiological descriptions of Taenia solium infection foci published, which are only recently rediscovered [21]. One was in an Orthodox Jewish community in New York, where 4 cases of neurocysticercosis were found around one Taenia solium carrier being a servant [22]. The second was observed during a mass treatment of 10.173 people in an Andean Ecuadorian Taenia solium endemic area [23]. The mean rate of the expulsion a tapeworm was 1.6\%. However, among 23 mountainous localities in 5 there were no $T$. solium carriers, and in another 5 localities between $7.4 \%$ and $21.1 \%$ of the inhabitants had expelled a tapeworm. Same farm and family agglomerations were generally observed. Following that study a definition of the $T$. solium focus has already been proposed and remains still open for discussion [1]:

a) A human T. solium tapeworm carrier [leaving her/him untreated is a medical error!];

b) A case of neurocysticercosis, especially that with a late epilepsy syndrome [a presence of a carrier in this case or in people living around is highly possible];

c) A farm producing cysticercotic pigs [a $T$. solium carrier is strongly suspected to be there];

d) A locality with the prevalence of pig cysticercosis over $5 \%$ [more than one carrier is usually there].

The immediate individual treatment is obligatory in any case of $T$. solium taeniosis as soon as it is detected. Daily production of over 100,000 eggs by a single tapeworm, is considering a high risk of the environment contamination for humans and pigs. The selective treatments are justified around a neurocysticercosis case and in a "cysticercotic" farm. Mass treatment may be justified in localities with high rate of pigs' cysticercosis. The effi- 
cacy of chemotherapeutic intervention has to be checkout after treatment and if not positive than repeated after 2 - 3 months.

Using focality syndrome of $T$. solium infections is important not only for the organized t/nc control programs but also in general medical (see points a and b) and veterinary practices (see points c and d). Successes in elimination small pox and some other viral infections by vaccination programs, by increasing the values of specific elimination "actions" had a negative impact on the importance of using a specific chemotherapy in the routine daily practices in elimination T. solium infections [1]. The notification of the neurocysticercosis and taeniosis cases has been proposed and somewhere implemented but it might be useful only if followed by an action of sanitary-epidemiological services, supervising treatments in the notified foci. This has been documented for Taenia saginata infections control studies in Poznan region (Poland) [5]. Proper training and professional education are needed to restore the important role, which medical and veterinary practicing doctors might be playing in the control of $\mathrm{t} / \mathrm{nc}$.

\section{Professional Training and Health Education Are Important in t/nc Control Activities}

The medical community at large is not being prepared to participate in prevention of t/nc. Teaching of parasitology in most of the Medical Faculties and paramedical schools never was a very strong issue. In particular, a tapeworm infection it is not regarded as a clinical problem, although it may cause a serious complication such as neurocysticercosis. The diagnosis of taeniosis is frequently overlooked and specific treatment not given, even in some neurological wards, where the prevention is traditionally left to veterinarians. The knowledge has to be promoted that leaving a T. solium carrier untreated is a medical error; looking for a carrier in a focus (e.g., around a neurocysticercosis or epilepsy case) should be a standard procedure but is not commonly accepted and practiced. Spreading these information has to be responsibility of medical teachers, bodies setting up medical standards and consultants responsible for the quality of medical and veterinary services. Promotion of such a professional knowledge is especially important among the primary health profession in endemic areas but it has to be supported by ensuring a local availability of taenicides in order to be useful. The successes in the control of lymphatic filariasis and soil-transmitted helminthiases are due to large free drugs donations making a high availability of effective anthelminthic; could a treatment of taeniosis be made so popular and easy? Taenia solium infections has its biological and economic cycles [24]. For rural poor breading the free roaming pigs does not need much investing except buying the piglets. The unlicensed pig and meat handlers are buying infected pigs cheaply and selling its meat at the normal price. The education can hardly work out where money and tradition are involved; proper legislation and supervision has to follow any health education to be effective. The t/nc education has made a great progress recently [25]. In education process, adopted to the local needs, the risk of acquiring neurocysticercosis and/or epilepsy has to be used as the primary motivation and the suggested preventive measures sometimes as simple as washing hands, when water is available. In t/nc control projects education needs a strong professional and financial backing.

\section{What We Need to Know to Improve and Push t/nc Control?}

There is a sort of financial chaos due to a lack of evident priorities and a lot of repetitions in the area of research related to the control of t/nc. Progress in basic research is frequently not implemented into the practice. Operational research in field is rare being more demanding to organize and perform than that at a laboratory room. The number of publications on clinical aspects of neurocysticercosis much exceeds the prevention issues [2]; in the clinical papers frequently preventive measures against spreading neurocysticercosis by fecal-oral route are even not mentioned.

There are some areas, where more information is needed to push the $\mathrm{t} / \mathrm{nc}$ control projects:

1) Standards of medical education are usually rather individual patients care oriented and less on public health matters. The results of the sporadic studies on the knowledge on some parasitic diseases among the absolvents of medical faculties are unsatisfactory. It would be useful to know how many medical doctors, e.g., specializing in mental health and neurology, know about the prevention of fecal-borne transmission of T. solium in neurocysticercotic patients and in the late epilepsy cases. The mental health units cannot concentrate on neurocysticercosis and epilepsy as a clinical conditions only but must be involved also in its prevention by diagnosing and treatment of concomitant taeniosis [2] [26]. The results might be used to make a pressure on universities to prepare 
better young doctors to practice the prophylactic measures. Editors of the academic textbooks have to be obliged to enlarge subchapters on prophylactics, including global control projects. International control programs against neglected diseases are not enough visible neither during the studies nor in the textbooks and medical press.

2) Support a larger production of the diagnostic tests on Taenia antigens in feces, or better in blood, and make it available at a low price, wherever are needed in $T$. solium endemic areas. The financial return to health sector would be in savings the expenses on the medical care of neurocysticercosis cases being prevented. Diagnosing human tapeworm carriers starts to be a burning issue in immigrant's and traveler's health problem.

3) Making a map of availability of taenicides in endemic areas and ensure that they are at hand, whenever needed; there is a precedence in implementation of official availability of the venom antiserum in the areas, where problems exist; why not for neurocysticercosis? Introduce a regular quality control of niclosamide, based on the size of active particles, and promote to respect its validation time. Clarify the frequency of neurological complications at using praziquantel in neurocysticercotic patients and make an official statement on the drug use in those cases.

4) Increase the health education on prevention of neurocysticercosis (also in urban areas) and try to offer free diagnostics and treatments of human taeniosis.

5) Find out whether the information from veterinary services about local pig cysticercosis prevalence is used for the $t / n c$ control purposes. Consider whether veterinarians should have a right to disseminate free teanicide in any $T$. solium foci.

These thoughts, putting the t/nc control problem in a wider perspective, are addressed to all medical professionals and health services managers, who are or become convinced that neglected neurocysticercosis deserve more attention and an effective implementation without much further delay [21] [27].

\section{Statement}

No financial support. No conflicts of interest.

\section{References}

[1] Pawlowski, Z.S. (2008) Control of Neurocysticercosis by Routine Medical and Veterinary Services. Transactions of the Royal Society of Tropical Medicine and Hygiene, 102, 228-232. http://dx.doi.org/10.1016/j.trstmh.2007.10.007

[2] World Health Organization (2015) Assembling a Framework for Intensified Control of Taeniosis and Neurocysticercosis Caused by Taenia solium. WHO, Geneva.

[3] Grove, D.I. (1990) A History of Human Helminthology. CAB International, Wallingford.

[4] Lightowlers, M.W. (2013) Control of Taenia solium Taeniosis/Cysticercosis: Past Practices and New Possibilities. Parasitology, 140, 1566-1577. http://dx.doi.org/10.1017/S0031182013001005

[5] Flisser, A., Wilms, K., Laclette, J.P., Larralde, C., Ridaura, C. and Beltran, F. (1982) Cysticercosis. Present State of Knowledge and Perspectives. Academic Press, New York.

[6] Schantz, P.M., Cruz, M., Sarti, E. and Pawlowski, Z. (1993) Potential Eradicability of Taeniosis and Cysticercosis. Bulletin of the Pan American Health Organization, 27, 397-403.

[7] (2003) International Task Force for Disease Eradication II. Carter Center, Atlanta.

[8] Flisser, A. and Jones, M.K. (2010) Neurocysticercocis May No Longer Be a Public Health Problem in Mexico. PLOS Neglected Tropical Diseases, 4, e831. http://dx.doi.org/10.1371/journal.pntd.0000831

[9] Alarcon, T.A. and Del Bruto, O.K. (2012) Neurocysticercosis: Declining Incidence among Patients Admitted to a Large Public Hospital in Guayaquil, Ecuador. Patogens and Global Health, 106, 310-311. http://dx.doi.org/10.1179/2047773212Y.0000000044

[10] Gilman, R.H., Gonzales, A.E., Llanos-Zavalaga, F., Tsang, V.C.W. and Garcia, H.H. (2012) Prevention and Control of Taenia solium Taeniosis/Cysticercosis in Peru. Pathogens and Global Health, 106, 312-318. http://dx.doi.org/10.1179/2047773212Y.0000000045

[11] Ito, A., Zhou, X.N., Craig, P.S. and Giraudoux, P. (2013) Control of Cestodezoonoses in Asia: Role of Basic and Applied Science. Parasitology, 140, 1547-1550. http://dx.doi.org/10.1017/S0031182013001157

[12] World Health Organization (2011) Report of the WHO Expert Consultation on Foodborne Trematode Infections \& Taeniosis/Cysticercosis. Vientiane, Lao People’s Democratic Republic, 12-16 October 2009, World Health Organization, Geneva.

[13] Lancet Editorial (2014) Neglected Tropical Diseases Becoming Less Neglected. Lancet, 383, 1269. 
http://dx.doi.org/10.1016/S0140-6736(14)60629-2

[14] Maurice, J. (2014) Of Pigs and People-WHO Prepares to Battle Cysticercosis. Lancet, 384, 571-572. http://dx.doi.org/10.1016/S0140-6736(14)61353-2

[15] Parise, M.E., Hotez, P.J. and Slutsker, L. (2014) Neglected Parasitic Infections in the United States: Needs and Opportunities. American Journal of Tropical Medicine and Hygiene, 90, 783-785. http://dx.doi.org/10.4269/ajtmh.13-0727

[16] Cantey, P.T., Coyle, C.M., Sorvillo, L.J., Wilkins, P.P., Starr, M.C. and Nash, T.E. (2014) Neglected Parasitic Infections in the United States: Cysticercosis. American Journal of Tropical Medicine and Hygiene, 90, 805-809. http://dx.doi.org/10.4269/ajtmh.13-0724

[17] Ito, A. (2013) Nothing Is Perfect! Trouble-Shooting in Immunological and Molecular Studies of Cestode Infections. Parasitology, 140, 1551-1565. http://dx.doi.org/10.1017/S0031182013000966

[18] Murrell, K.D. (2005) WHO/FAO/OIE Guidelines for the Surveillance, Prevention and Control of Taeniosis/Cysticercosis. OIE, Paris.

[19] Pawlowski, Z.S. (2006) Role of Chemotherapy of Taeniosis in Prevention of Neurocysticercosis. International Journal for Parasitology, 55, S105-S109.

[20] Pawlowski, Z.S. (1991) Control of Taenia solium Taeniasis and Cysticercosis by Focus-Oriented Chemotherapy of Taeniosis. The Southeast Asian Journal of Tropical Medicine and Public Health, 22, S284-S286.

[21] O’Neal, S., Winthrop, K. and Gonzales, A. (2011) Cysticercosis Control: Bringing Advances to the Field. Journal of Global Infectious Diseases, 3, 156-160. http://dx.doi.org/10.4103/0974-777X.81693

[22] Schantz, P.M., Moore, A.C., Munoz, J.L., et al. (1992): Neurocysticercosis in an Orthodox Jewish Community in New York City. The New England Journal of Medicine, 327, 692-695. http://dx.doi.org/10.1056/NEJM199209033271004

[23] Cruz, M., Davis, A., Dixon, H., Pawlowski, Z. and Proano, J. (1989) Operational Studies on the Control of Taenia solium Taeniasis/Cysticercosis in Ecuador. Bull World Health Organ, 67, 401-407.

[24] Pawlowski, Z. (1994) Taeniasis and Cysticercosis. In: Hui, Y.H., Gorham, J.R., Murrell, K.D. and Cliver, D.O., Eds., Foodborne Diseases Handbook, Vol. 2, Marcel Dekker Inc., New York, 199-254.

[25] Johansen, M.V., Trevisan, C., Braae, U.C., Magnussen, P., Ertel, R.L., Mejer, H. and Saarnak, C.F.L. (2014) The Vicious Worm: A Computer-Based Taenia solium Education Tool. Trends in Parasitology, 30, 372-374. http://dx.doi.org/10.1016/j.pt.2014.06.003

[26] Winkler, A.S. (2012) Neurocysticercosis in Sub-Saharan Africa: A Review of Prevalence, Clinical Characteristics, Diagnosis and Management. Pathogens and Global Health, 106, 261-274. http://dx.doi.org/10.1179/2047773212Y.0000000047

[27] Pawlowski, Z., Allan, J. and Sarti, E. (2005) Control of Taenia solium Taeniosis/Cysticercosis: From Research towards Implementation. International Journal for Parasitology, 35, 1221-1232. http://dx.doi.org/10.1016/j.ijpara.2005.07.015 